Cahiers « Mondes anciens »

ANCIENS

Histoire et anthropologie des mondes anciens

4 | 2013

Journées doctorales ANHIMA 2010 et 2011

\title{
Le passage de Célestius à Carthage : un moment clé du pélagianisme
}

\section{Zohra Picard-Mawji}

\section{OpenEdition}

\section{Journals}

Édition électronique

URL : http://journals.openedition.org/mondesanciens/1003

DOI : 10.4000/mondesanciens. 1003

ISSN : 2107-0199

\section{Éditeur}

UMR 8210 Anthropologie et Histoire des Mondes Antiques

Référence électronique

Zohra Picard-Mawji, «Le passage de Célestius à Carthage : un moment clé du pélagianisme », Cahiers «Mondes anciens » [En ligne], 4 | 2013, mis en ligne le 01 juillet 2013, consulté le 05 mai 2019. URL http://journals.openedition.org/mondesanciens/1003; DOI : 10.4000/mondesanciens. 1003

Ce document a été généré automatiquement le 5 mai 2019.

\section{(c) (i) () $\Theta$}

Les Cahiers «Mondes Anciens » sont mis à disposition selon les termes de la licence Creative Commons Attribution - Pas d'Utilisation Commerciale - Pas de Modification 4.0 International. 


\title{
Le passage de Célestius à Carthage : un moment clé du pélagianisme
}

\author{
Zohra Picard-Mawji
}

\section{Introduction}

1 «Ce fut Célestius, et non pas Pélage, qui déchaîna la crise en Afrique. Dès son arrivée à Carthage, il était intervenu résolument dans les débats en cours qui, semble-t-il, roulaient sur l'éternel mystère de l'origine de l'âme ». C'est par ces mots que Peter BRown (2001, p. 453) introduit, pour la première fois dans sa célèbre biographie de saint Augustin, le personnage de Célestius. Celui-ci est tout de suite lié à « l'Afrique » et à la «crise » qu'il aurait apparemment déclenchée au cours de conversations sur « l'origine de l'âme », l'un des grands sujets théologiques du moment ${ }^{1}$, intéressant les pélagiens au plus haut point. Pourquoi?

2 Il convient tout d'abord d'expliquer brièvement ce qu'est le pélagianisme ${ }^{2}$. Le mot est formé sur le nom de Pélage, un chrétien breton ${ }^{3}$ ayant vécu entre la fin du IV et le début $\mathrm{du} \mathrm{v}^{\mathrm{e}}$ siècle et dont le mode de vie ascétique rappelle celui d'un moine. Il se distingue à son époque par la profession d'une exaltation du libre-arbitre selon laquelle l'homme peut se sauver par ses forces propres. Ainsi pour les pélagiens, la nature humaine n'est pas viciée par les effets du péché originel et, par conséquent, l'homme peut être parfait dès cette vie terrestre. On comprend alors l'intérêt que les pélagiens portent à la question de l'origine des âmes telle qu'elle était posée en Afrique. En effet, depuis Tertullien, on y penchait en faveur du traducianisme c'est-à-dire du fait que les âmes se perpétuent par voie de génération, par «passeur » (tradux) ou «transmetteur interposé » (per traducem). De cette façon, la corruption qui a touché Adam après le péché originel s'est transmise héréditairement à ses descendants, c'est-à-dire à tout le genre humain, ce qui ruine évidemment le principe de la nature parfaite de l'homme qui constitue la base du pélagianisme. 
3 C'est ainsi que Célestius ${ }^{4}$ s'est illustré dès son arrivée en Afrique par son hostilité au traducianisme, attirant la suspicion sur ceux qui suivaient Pélage et dont il était sûrement le premier disciple. À travers lui, ce serait donc la pensée du moine breton qui s'exprimerait, comme l'expose Augustin dans son De gratia Christi et de peccato originali :

Attendite itaque quid Caelestius apertissime dixerit, et ibi uidebitis quid uobis Pelagius occultauerit. (...) Hunc sensum suum uobis aperire Pelagius uel timuit uel erubuit, quem discipulus eius palam sine ullius obscuritatis ambagibus apud sedem apostolicam profiteri nec timuit nec erubuit.

(Aug., Gr. et pecc. or., 6, 6)

C'est pourquoi soyez attentifs à ce que Célestius a dit, et là vous y verrez ce que Pélage vous a dissimulé (...). Cette pensée qui est la sienne, Pélage a craint ou a eu honte de vous en faire part, mais son disciple n'a ni craint ni eu honte de la professer au grand jour devant le Siège apostolique, sans détours d'une quelconque obscurité.

4 Célestius est, en effet, partisan du créatianisme, selon lequel Dieu crée l'âme de tous les êtres humains au fur et à mesure de leur conception. Il profite donc de ce débat à Carthage pour introduire ses idées nouvelles sur la condition humaine en général, et celles-ci entraînent à leur tour une vision neuve des moyens du salut.

Voilà déjà un certain temps que Pélage répand ses idées à Rome, puisque selon Marius Mercator, lorsque Célestius débarque en Afrique en $410 / 411^{5}$, il y a déjà 20 ans qu'il est disciple de Pélage ${ }^{6}$. Et pourtant depuis les années 390, les idées pélagiennes, si elles ont pu déclencher des débats ${ }^{7}$, n'ont jamais attiré défavorablement l'attention des autorités ecclésiastiques ou impériales et encore moins donné lieu à une quelconque condamnation. La « crise », pour reprendre le terme de Peter Brown, commence bien en Afrique, à Carthage, et à la suite de cette prédication de Célestius. Ce qui fait bien de l'année 411 une « année cruciale » comme la qualifie Walter DUNPHY (2005, p. 391). Et c'est pourquoi il nous a paru intéressant de nous arrêter sur ce moment bien précis dans le cadre d'une journée consacrée à l'importance du passage : lorsque la figure du " passeur » qu'est Célestius (des idées de Pélage) « passe » à Carthage en 411, il s'agit véritablement d'un moment-clé qui modifie de façon irréversible l'histoire du pélagianisme, le faisant « passer » dans une autre phase de son histoire, et conduit Augustin d'Hippone et l'Église catholique à définir certains points de doctrine avec davantage de précisions.

On ne peut que rappeler la difficulté à traiter ce sujet vu la rareté des sources, problème particulièrement évident pour les spécialistes de l'histoire des doctrines. Le pélagianisme a, en effet, été condamné du vivant de ses concepteurs et par conséquent la transmission des textes pélagiens a été vite interrompue. Comme le pélagianisme est surtout connu grâce à ce qu'en relatent les antipélagiens, l'historien doit sans cesse se méfier de la subjectivité latente des sources dont il dispose. En raison de tous ces éléments, certaines données exposées ici ne sont que des hypothèses, parfois âprement débattues.

Cette mise en garde faite, nous pouvons nous attacher plus précisément à l'étude de ce passage de Célestius à Carthage en suivant une trame chronologique. L'étude des conditions de l'arrivée de Célestius à Carthage nous permet de nous intéresser aux conditions matérielles de son voyage ainsi qu'aux débuts de sa prédication en Afrique (I). Celle-ci déclenche débats et réactions en Afrique (II) puis dans tout le monde romain, éléments qui marquent de façon définitive l'histoire du pélagianisme (III). 


\section{L'arrivée de Célestius à Carthage}

8 Un certain flou entoure les circonstances de l'arrivée de Célestius à Carthage ainsi que les conditions dans lesquelles il a pu faire entendre son point de vue sur l'origine des âmes. Nous allons tenter de les éclaircir en proposant une hypothèse sur le chemin emprunté par Célestius pour traverser la Méditerranée de Rome à Carthage, avant de nous intéresser aux débuts de sa prise de parole dans la capitale africaine. Nous commencerons, tout d'abord, par dresser un portrait de Célestius.

\section{Portrait de Célestius ${ }^{8}$}

9 Célestius est décrit par ses contemporains comme quelqu'un d'intelligent - Augustin parle ainsi d'un "homme à l'intelligence si pénétrante ${ }^{9}$ " - aux qualités oratoires certaines. Sans doute aristocrate de naissance, il suit des études de droit, améliorant ainsi sa «loquacitas» naturelle selon Marius Mercator ${ }^{10}$. Ces éléments de personnalité lui permettent de jouer un rôle particulier : celui de passeur. Il est qualifié par les historiens d'« animateur » du réseau pélagien, « d'organisateur doué pour la propagande » (PIETRI 2005, p. 460), qui « n'avait pas la prudence ni la modération de son maitre » (LANCEL 1999, p. 460) ce qui le faisait passer pour un « aristocrate excentrique » (BROWN 1970, p. 65). Et c'est à Carthage qu'il semble commencer son œuvre de passeur, de tradux des idées de Pélage. Comment est-il arrivé à Carthage?

\section{De Rome à l'Afrique}

10 Pélage et Célestius partageaient à Rome la compagnie d'aristocrates chrétiens ${ }^{11}$ attirés par l'ascétisme, notamment la grande famille des Valerii (BROWN 1970). 
Essai de reconstitution du voyage de Célestius

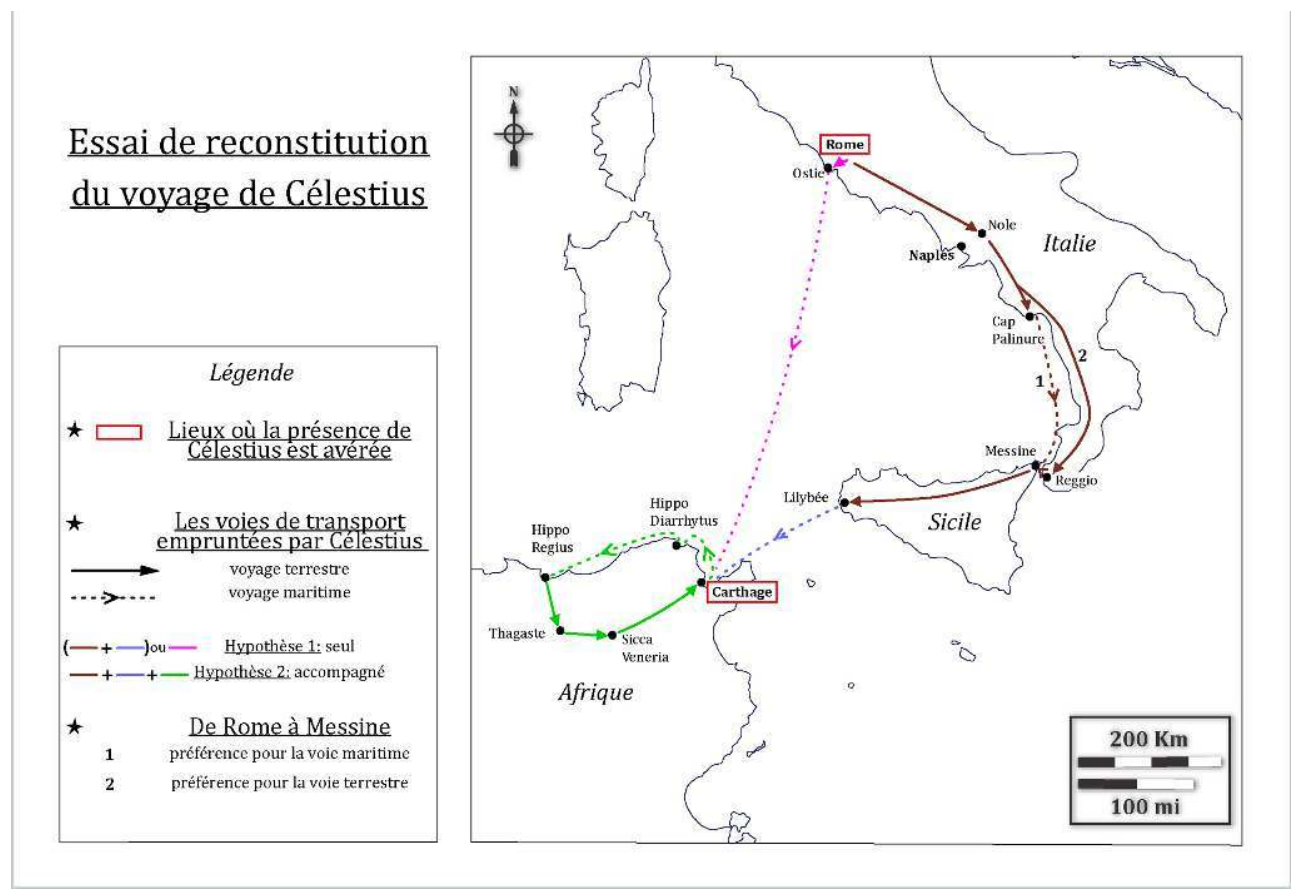

Z. Picard

On peut de ce fait avancer sans trop d'hésitation que Pélage a suivi les Valerii ${ }^{12}$ dans leur exil, provoqué par la menace des Wisigoths en Italie en 409-410, à savoir Albine, sa fille Mélanie la Jeune et le mari de cette dernière Pinianus ${ }^{13}$. Mais quid de Célestius ? Nous n'avons en effet aucune trace de lui jusqu'à 411 . Notre hypothèse est simple: il a vraisemblablement voyagé avec Pélage, qui accompagnait lui-même sans doute les Valerii. On peut suivre cet itinéraire de voyage, reconstitué à partir des sources littéraires disponibles et des grandes routes en usage à cette époque (selon la synthèse d' ARNAUD 2005), sur la carte Essai de reconstitution du voyage de Célestius (cf. carte). La première étape du voyage est la cité de Nole, rejointe par la via Appia à partir de Rome, où les Valerii retrouvent l'évêque Paulin ${ }^{14}$. Puis, ils gagnent la Sicile et enfin l'Afrique. Pour la suite du voyage, nous disposons de deux informations qui nous permettent d'émettre une hypothèse assez sûre. La première est que Pélage est à Hippone à la fin de l'année $410^{15}$ alors qu'Augustin en est absent (PERLER 1969, p. 281). La seconde est le fait que les Valerii sont accueillis à Thagaste par l'évêque de la cité, Alypius ${ }^{16}$. Or Hippone était l'un des ports africains les plus importants - et il est le plus près de Thagaste. Il semble donc que les Valerii aient débarqué directement à Hippone depuis la Sicile, ou en effectuant peut-être une escale de ravitaillement à Carthage. On peut donc supposer - en l'absence totale de sources - que Célestius est arrivé à Hippone à l'automne 410 - avant la mi-novembre, date à laquelle la saison de la navigation est terminée - et qu'il a passé l'hiver à Thagaste ${ }^{17}$ comme ses protecteurs.

Au cours de ce voyage, les deux pélagiens sont toujours restés dans un environnement familier, entourés d'aristocrates chrétiens attirés par l'ascétisme ${ }^{18}$. C'est sans doute à la fin de l'hiver ou au cours du printemps qu'ils quittent ce monde isolé pour se rendre à Carthage, où la présence de Pélage est attestée en mai $411^{19}$. Comme l'écrit Serge LANCEL 
(1999, p. 458) : «Pélage se hâta de rejoindre Carthage où il savait pouvoir retrouver une partie de cette noblesse chez qui il avait ses entrées à Rome ».

Célestius a aussi pu voyager seul, sans pour autant arriver trop tôt en Afrique, ou alors en y restant très discret à propos de ses idées. On préférera cependant la première solution où l'on situe un séjour en Sicile au cours duquel il aurait fait beaucoup d'adeptes ${ }^{20}$ même si certains chercheurs, comme Guido HONNAY (1994, p. 274), rejettent cette possibilité. À cette époque encore, Célestius est un parfait inconnu: les contemporains n'ont donc aucune raison de noter ses faits et gestes - ce qui peut expliquer bien des choses contrairement à Pélage ${ }^{21}$ qui avait déjà fait parler de lui outre-mer. Ces circonstances changent après son passage à Carthage où ses discours font basculer les idées pélagiennes dans une phase d'expansion active. Nous sommes bien ici à un moment-clé de l'histoire du pélagianisme.

Quant à Pélage, il ne reste pas très longtemps à Carthage et en semble parti avant même l'ouverture de la conférence contre les donatistes, le $1^{\text {er }}$ juin $411^{22}$. Plusieurs hypothèses ${ }^{23}$ peuvent être émises pour expliquer ce départ quelque peu précipité, semble-t-il. Pélage at-il voulu éviter les conflits qui se dessinaient entre Célestius et ceux qu'il contredisait? Ou bien a-t-il tout simplement voulu continuer son voyage jusqu'en Terre sainte, lieu qui constituait peut-être dès le départ le but de son périple. En ce lieu, il pourrait s'attacher de nouveaux disciples. Plus probablement encore, c'est ce faisceau de causes qui pousse Pélage à quitter l'Afrique et à se séparer de Célestius.

\section{Début des discussions à Carthage}

15 Le pélagianisme n'avait jusque-là touché que quelques aristocrates italiens et surtout romains. Or les discours de Célestius connaissent une audience importante ${ }^{24}$, assurant ainsi une diffusion assez large de ses idées à Carthage. Dans quelles circonstances? Rappelons que c'est un laïc et que le prêche dans les églises lui est donc interdit car réservé aux évêques, parfois aux prêtres. Il n'est pas d'usage non plus que les chrétiens prêchent sur les places publiques. Cependant, on ne peut exclure cette possibilité si l'on se réfère à Vincent de Lérins qui écrit à propos des hérétiques - sans viser nommément les pélagiens :

Siue enim apud suos siue alienos, siue priuatim siue publice, siue in sermonibus siue in libris, siue in conuiuiis siue in plateis, nihil umquam paene de suo proferunt quod non etiam scripturae uerbis adumbrare conentur.

(Vincent de Lérins, Commonitorium, 25, 2)

Que ce soit auprès des leurs ou auprès des étrangers, dans le privé ou en public, dans leurs propos ou dans leurs livres, dans les repas ou sur les places publiques, ils n'allèguent presque rien de leur cru qu'ils ne s'efforcent de l'obscurcir avec des paroles de l'Écriture.

Célestius et son maître, qui fréquentaient des cercles de l'aristocratie romaine chrétienne à Rome comme on l'a vu, se sont naturellement retrouvés dans des groupes de discussions parmi les émigrés d'Italie, qui perpétuaient la sociabilité des exilés de Rome. Walter DUNPHY $(2009$, p. 97) va jusqu'à dire que certains membres de ces groupes ont pris la défense de Célestius face aux attaques dont il a été la cible, à cause d'une sorte de solidarité sociale ${ }^{25}$. Ce sont les premiers auditeurs que Célestius touche et c'est sur eux qu'il s'appuie pour faire circuler ses idées. Car parmi ceux-ci, il se trouvait certainement des Africains, liés d'une façon ou d'une autre aux exilés - membres de l'aristocratie ou du clergé carthaginois - qui ont pu faire la jonction avec une partie de la population de la 
capitale africaine, gagnée à son tour aux idées du pélagien. Célestius dispose ainsi d'un réseau qui lui ouvre l'accès à une population inconnue et importante numériquement. En effet, il n'est pas tout seul à répandre ses idées (Augustin parle de plusieurs adversaires dans le Pecc. mer. $)^{26}$ même si la postérité n'a retenu que son nom.

Nous pouvons citer une personne au moins qui a pu assister à ces discussions : le tribun et notaire Marcellinus, mandaté par l'empereur Honorius pour mener les débats de la conférence de Carthage de 411. Grâce à celle-ci, Marcellinus avait été témoin des connaissances théologiques comme des qualités de polémiste d'Augustin ; c'est donc tout naturellement vers lui que le fonctionnaire impérial se tourne, afin de connaître l'orthodoxie des discussions impliquant Célestius. Il met ainsi Augustin d'Hippone au courant de la tenue de débats quotidiens, ce qui nous permet d'en tirer un précieux élément de datation. L'attention d'Augustin n'ayant pas été attirée par Célestius lors de la conférence de Carthage, nous en déduisons que les fameuses discussions ont commencé après le 8 juin 411 et qu'elles ont duré un certain temps comme nous le prouve le De gestis Pelagii : "Marcellinus (...) subissait alors chaque jour l'assaut de ces polémistes fort importuns ${ }^{27}$ », entretenant une «lutte (...) si violente, intense et sans répit ${ }^{28}$. Cette dernière phrase nous permet de conclure de façon certaine que Marcellinus n'a pas simplement été mis au courant de ce qui se passait, mais qu'il était bien présent lors des rencontres avec Célestius ou ses partisans.

Bien que le nom de Célestius ne soit cité nulle part, la réponse d'Augustin à la lettre perdue de Marcellinus, c'est-à-dire le De Peccatorum meritis et remissione, nous permet d'avoir une vue d'ensemble des thèmes abordés, qui mettent par la suite Célestius dans une situation difficile. On complètera les informations issues de cet échange grâce à plusieurs traités du docteur d'Hippone. L'une des difficultés du corpus utilisé réside en ce qu'il est composé de sources postérieures - voire très postérieures - aux événements ${ }^{29}$.

\section{Le débat et ses conséquences immédiates}

19 À l'été 411, l'Église d'Afrique pense tourner la page de la crise terrible qu'elle traversait depuis près d'un siècle. Et voilà que, dans la bonne société carthaginoise, résonnent des idées nouvelles, dérangeantes, voire dangereuses. Lesquelles?

\section{Les idées de Célestius ${ }^{30}$}

Rappelons que Célestius profite du débat sur l'origine des âmes pour développer des points de la doctrine pélagienne. Le créatianisme permet de conclure que la nature humaine n'est pas viciée par le péché originel. Dans ce cas, la rémission des péchés obtenue par le sacrement du baptême ne concerne que les péchés personnels, et non la faute d'Adam qui n'a corrompu personne d'autre que le premier homme. Selon ce raisonnement, il devient alors inutile de pratiquer le baptême des nouveaux-nés puisqu'ils n'ont encore commis aucun péché. Ces thèmes sont développés d'autant plus facilement qu'ils ne font pas l'objet d'une doctrine clairement arrêtée par l'Église catholique. N'appartenant pas au dogme, ils peuvent donc faire l'objet de débats. Ces thèmes tiennent d'ailleurs une place très importante pendant toute la controverse pélagienne, développés tout particulièrement par Célestius comme nous l'apprend le Praedestinatus ${ }^{31}$, renforcant par là même la conviction d'attribuer à Célestius le rôle primordial dans le débat carthaginois. Son passage dans la capitale africaine n'est donc 
pas seulement un moment-clé du pélagianisme, mais aussi du développement de la doctrine catholique puisque cette nécessité ou non du baptême des enfants est débattue jusque dans les années 420 , c'est-à-dire jusqu'à la fin de la controverse impliquant Augustin et Julien d'Éclane ${ }^{32}$.

Ce sont toutes ces questions que Marcellinus porte à l'attention d'Augustin d'Hippone dans une lettre qui n'est pas parvenue jusqu'à nous mais dont nous connaissons la teneur grâce à la réponse de l'évêque sous la forme d'un traité: le De Peccatorum meritis et remissione et de baptismo paruulorum, qui traite comme son nom l'indique du salaire du péché - à savoir la mort - de la façon de racheter ce péché, ainsi que de la nécessité de baptiser les enfants. Augustin n'y cite pas Célestius ; il semble pourtant improbable qu'il ne sache pas déjà de qui il s'agit - sans forcément connaître son nom - par exemple lorsqu'on lit la phrase suivante :

Proinde quod adtinet ad baptismum paruulorum, ut eis sit necessarius, redemptionem etiam ipsis opus esse concedunt, sicut cuiusdam eorum libello breuissimo continetur, qui tamen ibi remissionem alicuius peccati apertius esprimere noluit.

(Aug., Pecc. mer., 34, 63)

Aussi bien, quand il s'agit du baptême des petits enfants, afin que ce sacrement leur soit nécessaire, ils admettent que ces derniers ont besoin d'une rédemption, comme on peut le lire dans un court écrit de l'un d'entre eux, qui cependant ne veut pas s'y exprimer plus ouvertement sur la rémission des péchés. immédiate que Célestius rencontre à Carthage en 411. Tout d'abord, on peut rappeler que les Africains sont très sensibles à la question du baptême puisque c'est l'un des enjeux de la crise donatiste ; ceux-ci en effet rebaptisaient les catholiques qui les rejoignaient contrairement à l'usage en vigueur chez ces derniers. Or la conférence de Carthage de juin 411 avait fait renaître l'espoir d'une unité possible de l'Église d'Afrique. On peut donc imaginer le trouble engendré par l'idée que le baptême n'est pas indispensable au salut, idée importée par ces Romains en exil, alors qu'il y a encore sûrement dans la capitale africaine une grande part du public de la conférence, dont de nombreux ecclésiastiques. Cette donnée explique sans doute l'audience importante de Célestius, tout autant d'ailleurs que la ferme réaction des Africains qui rejettent la possibilité d'amoindrir le caractère rédempteur du baptême. Augustin insiste sur ce point: "Les chrétiens d'Afrique ont parfaitement raison de dire que le baptême précisément n'est rien d'autre que le salut ${ }^{34} »$. Les Romains ${ }^{35}$ sont donc vus ici comme des novateurs ayant rencontré l'opposition des Africains. Pourtant, ce n'est pas d'eux que vient l'attaque la plus forte contre Célestius, mais d'un autre émigré italien. 


\section{La réunion de Carthage}

lettre 157 d'Augustin à Hilaire de Syracuse, le De gestis Pelagii ou encore le De gratia Christi et de peccato originali, datant respectivement de $414,416-417$ et 418 . On y apprend que les propos «nouveaux ${ }^{36}$ » de Célestius ne déclenchent pas encore de mesures officielles. Les détracteurs de Célestius sont alors des individus isolés et le combat reste au seul niveau des idées. Il semble donc que les idées novatrices de Célestius seraient passées quasiment inaperçues si celui-ci n'avait pas eu "l'imprudence » (LANCEL 1999, p. 460) de formuler la demande d'être ordonné prêtre dans le clergé de Carthage. Avait-t-il dès le début cette intention et donc la volonté de s'installer vraiment en Afrique, et non pas d'y faire un simple passage ? Il semble plutôt que le but d'une telle démarche était d'obtenir un titre qui lui assure une autorité certaine en matière de doctrine. Ce titre, allié à son mode de vie ascétique, lui assurait de pouvoir répandre ses idées plus facilement, à la fois physiquement - accès à des espaces de paroles fermés aux laïcs, à un auditoire important comme dans le cadre de l'enseignement catéchétique - mais aussi moralement grâce à l'autorité associée à la condition de prêtre. C'est pourquoi, le refus carthaginois ne décourage pas Célestius d'accéder à la prêtrise (il l'obtient par la suite à Éphèse).

La candidature de Célestius l'expose ainsi au grand public, donnant un nouvel écho à ses idées inédites. C'est un certain Paulin, diacre de l'Église de Milan ${ }^{37}$, alors présent en Afrique en qualité d'administrateur des biens de son Église dans la province, qui s'oppose alors frontalement au pélagien et se charge de l'accuser auprès du clergé de Carthage ${ }^{38}$. Deux hypothèses peuvent être proposées pour tenter d'expliquer cette situation :

- Paulin connaissait déjà Célestius et avait pour lui une inimitié ancienne en raison de ses idées, dont il aurait entendu parler alors soit à Milan ${ }^{39}$, soit lors de son passage à Rome ${ }^{40}$.

- Une autre hypothèse, défendue par Otto Wermelinger $(1975, \text { p. } 8)^{41}$, considère que Paulin était un "homme de paille »: le diacre milanais aurait été poussé à porter une accusation contre Célestius par des gens haut placés, qui «tirent les ficelles ». Il n'y a pas de preuves permettant de prouver cette thèse. On pourra cependant remarquer que ceux qui saisissent les autorités ecclésiastiques contre les pélagiens sont bien souvent des étrangers au lieu ${ }^{42}$.

on ne connaît donc pas les motivations exactes qui poussent Paulin à donner un tournant judiciaire à la candidature de Célestius à la prêtrise, ni la date précise de la réunion de Carthage qui examine les chefs d'accusation portés contre le pélagien. Cette question est même l'objet d'âpres débats ${ }^{43}$.

Bien qu'une date antérieure à la conférence ait pu être proposée pour la réunion de Carthage (коормANs 1954, р. 150), nous penchons pour une date postérieure au 8 juin 411. Lors de cette réunion, Augustin n'est pas ou plus présent à Carthage. Elle n'a donc pu se dérouler avant le 29 juin 411, date à laquelle l'évêque d'Hippone est encore dans la capitale, puisqu'il y prononce un sermon ${ }^{44}$. Il est d'ailleurs encore présent à la miseptembre ${ }^{45}$, mais rien ne prouve qu'il ait passé tout l'été à Carthage.

Après avoir déterminé le terminus post quem, il convient de s'intéresser au terminus ante quem. On exposera pour cela une donnée souvent laissée de côté, qui est celle du voyage de Célestius vers l'Orient et des indicateurs chronologiques qu'il peut nous fournir ${ }^{46}$. La lecture du Commonitorium super nomine Caelestii de Marius Mercator (Coll. Palatina 36, ACO I, 5,1, p. 66, 1. 22-25) nous permet de penser que Célestius s'embarque pour l'Orient très peu de temps après la réunion de Carthage pour un voyage qui a sans doute duré au moins 
une dizaine de jours ${ }^{47}$, et peut-être bien plus s'il a été ponctué de plusieurs escales importantes. Or la saison de la navigation s'arrêtant mi-novembre - et celle de la bonne navigation le 14 septembre - Célestius a dû partir au plus tard début novembre et sans doute un peu plus tôt, sans qu'il soit possible d'affiner encore la date. Avant cela, il a fallu qu'il se fasse connaître, que ses idées soient assez répandues, qu'il demande à être intégré au clergé de Carthage, que Paulin de Milan lance une procédure contre lui et qu'il soit convoqué pour plusieurs séances devant des évêques qui devaient le juger. On imagine aisément que Célestius participe à des débats informels tout au long de l'été et Augustin l'a peut-être même entendu de ses propres oreilles puisqu'il déclare :

Nam ante paruum tempus a quibusdam transitorie conloquentibus cursim mihi aures perstrictae sunt, cum illic apud Carthaginem essemus, "non ideo paruulos baptizari, ut remissionem accipiant peccatorum, sed ut sanctificentur in Christo".

(Aug., Pecc. mer., III, 6, 12.)

En effet, il y a peu de temps, alors que nous étions là à Carthage, mes oreilles ont été, comme en courant, frappées par certaines personnes qui s'entretenaient en passant : “Ce n'est pas pour cette raison que les petits enfants sont baptisés, à savoir afin qu'ils obtiennent la rémission de leur péché, mais afin qu'ils soient sanctifiés dans le Christ".

L'expression "ante paruum tempus ", comme le fait bien remarquer François REFOULÉ (1963, p. 43), suggère qu'Augustin fait référence à la fin de son séjour à Carthage donc à la mi-septembre. Pourquoi n'a-t-il pas dès lors engagé le débat avec Célestius ? Il répond :

Qua nouitate permotus et quia opportunum non fuit, ut contra aliquid dicerem, et non tales homines erant, de quorum essem auctoritate sollicitus, facile hoc in transactis atque abolitis habui. Et ecce iam studio flammante defenditur, ecce scribendo etiam memoriae commendatur, ecce res in hoc discrimen adducitur, ut hinc etiam a fratribus consulamur, ecce contra disputare atque scribere cogimur!

(Aug., Pecc. mer., III, 6, 12.)

Bien qu'étonné par cette nouveauté mais parce qu'il n'était pas opportun que je la contredise et parce que ce n'était pas des hommes connus dont l'autorité aurait pu m'inquiéter, j'ai facilement tenu celle-ci pour terminée et abolie. Et voici qu'on la défend maintenant d'un zèle ardent, voici qu'on perpétue même sa mémoire par l'écriture, voici que l'affaire est conduite à un tel point que nous sommes même consulté par nos frères, voici que nous sommes poussé à disputer et écrire contre elle!

31 Si Augustin a pu être poussé à combattre dès cette époque les idées pélagiennes au travers de sermons et conférences, comme il l'indique dans ses Retractationes (II, 33), il n'a en revanche pas jugé utile de réagir contre Célestius car celui-ci n'avait à l'époque aucune légitimité et n'était donc pas susceptible d'avoir de l'influence sur quiconque. Sa force de persuasion n'avait ainsi pas été correctement évaluée. De plus, dans son dernier sermon prononcé à Carthage, Augustin ne fait nulle mention d'une quelconque mesure disciplinaire envers un certain Célestius ce qui va dans notre sens de dater la réunion de Carthage après le départ d'Augustin pour Hippone, soit postérieurement au 14 septembre 411.

Ainsi, Célestius a demandé son ordination après la mi-septembre - qualité qui lui aurait conféré une auctoritas indiscutable - ce qui déclenche la réaction de Paulin puis aboutit à l'organisation d'une réunion du clergé de Carthage que nous estimons avoir eu lieu aux alentours d'octobre 411.

La nature de cette réunion est, elle aussi, objet de discussion. S'agit-il d'une réunion locale, d'un concile - et dans ce cas avec quels évêques? Et quelle en a été l'issue ? : une simple remontrance, une vraie condamnation - voire l'excommunication de Célestius? Il 
semble qu'elle se soit déroulée en plusieurs séances ${ }^{48}$ et qu'il y ait eu plusieurs évêques sous la présidence d'Aurélius de Carthage. La présence de ces évêques ne permet pas de confirmer la tenue d'un concile formel; il s'agit peut-être d'évêques simplement de passage, notamment si l'on situe le concile à l'été ou automne 411 à savoir juste après la conférence qui avait rassemblé plus de 500 évêques (donatistes et catholiques). Il peut aussi s'agir d'évêques de cités voisines, venus en peu de temps à la demande d'Aurélius. Il est difficile de répondre à ces questions car il est peu aisé de faire la part entre les faits qui se sont réellement déroulés et la mémoire de cette réunion.

\section{La mémoire du passage de Célestius à Carthage}

Les sources postérieures sont de fait très marquées par la mémoire de leurs auteurs. Par ailleurs, le contexte polémique de lutte contre une hérésie est un facteur de subjectivité à prendre en compte, d'autant plus que les enjeux sont très importants. On constate ainsi à la lecture des sources que le sort de Célestius à l'issue de la réunion de Carthage n'est pas toujours clairement défini, même si les antipélagiens la considèrent comme un précédent incontestable. Le passage de Célestius à Carthage et ses conséquences marquent ainsi de façon indélébile l'histoire du pélagianisme.

\section{Le sort de Célestius à l'issue de la réunion de Carthage}

Le sort de Célestius est donc incertain. Le De peccatorum meritis de 411-412 ne fait ainsi nulle mention d'un procès, Augustin ne semblant pas même être au courant de la procédure qui a été mise en place. Il affirme simplement que le processus enclenché a permis aux novateurs de modifier leur pensée dans le sens de leurs contradicteurs ${ }^{49}$. Célestius a, en effet, admis la nécessité du baptême des enfants, concession qu'il consigne dans un libellus defensionis. La source le plus proche chronologiquement de la réunion de Carthage est donc très évasive et va dans le sens d'une reprise fraternelle plutôt que d'un procès contre un potentiel hérétique.

La teneur n'est plus du tout la même deux ans plus tard, en 414, alors que Célestius est de nouveau mis en cause par différentes personnes dans plusieurs provinces. Augustin parle cette fois-ci d'un «jugement épiscopal» (Ep. 157, 3, 22 « iudicium episcopale »), qui indiquerait la tenue d'un vrai procès, insistant plus sur l'aspect disciplinaire de la résolution du cas Célestius. L'attitude du docteur d'Hippone a vraiment changé, ce que traduit l'abandon des termes évasifs du De peccatorum comme « cuiusdam $»^{50}$ pour exposer directement le nom de Célestius. En effet, Augustin juge que celui-ci est alors une menace sérieuse pour les chrétiens et qu'il convient donc d'exposer son nom. C'est peut-être aussi une façon d'appliquer une peine supplémentaire à Célestius puisqu'Augustin juge que la réunion de Carthage s'est résolue trop vite et sans sanction majeure semble-t-il :

Sed quia magis conuictus et ab ecclesia detestatus quam correctus et pacatus abscessit, ueritus sum, ne forte ipse ibi sit, qui uestram fidem perturbare conatur; ideo nominis ipsius commemorationem putaui esse faciendam.

(Aug. Ep. 157, 3, 22)

Mais parce qu'il s'en est sorti, bien plutôt avec la réfutation et les blâmes de l'Église qu'après rétractation et absolution, je crains que ce ne soit encore lui qui soit dans votre pays en train d'essayer de troubler votre foi, c'est pourquoi j'ai cru devoir mentionner son nom. 


\section{"confondu " et que le "synode africain a prononce une imprécation " contre lui.} Célestius a alors été obligé de se repentir ${ }^{51}$.

Une étape supplémentaire est franchie dans les récits les plus tardifs. Quelques années plus tard, en 427, Augustin dans les Retractationes ${ }^{52}$ présente Célestius comme explicitement excommunié tandis que Marius Mercator le décrit comme "privé de la communion de l'Église ${ }^{53}$ ». L'avis des chercheurs sur cette question est partagé ét $^{54}$. Cette décision n'alarme cependant pas le pélagien qui quitte l'Afrique vraisemblablement peu après le jugement ${ }^{55}$; il semble avoir annoncé faire appel à Rome de la décision ${ }^{56}$, quelle qu'elle soit, prise par l'assemblée de Carthage, peut-être parce qu'il estime que, en tant que non africain, la juridiction africaine n'est pas compétente pour le juger ou qu'il compte sur la bienveillance romaine à son sujet (voir supra). Célestius continue alors à diffuser les idées pélagiennes dans d'autres parties de l'Empire, mais nous dépassons ici les cadres géographique et chronologique de notre propos.

\section{L'ancrage du pélagianisme et l'aura de Célestius}

C'est donc bien grâce à ce passage à Carthage que Célestius s'est non seulement fait connaître mais s'est aussi imposé comme l'un des penseurs du pélagianisme. Il convient donc de bien lui redonner la place qu'il mérite dans la controverse pélagienne ${ }^{57}$. Il reste par ailleurs très actif par la suite ${ }^{58}$, acquérant ainsi le privilège - et, dans le cas d'espèce, aussi le déshonneur - de voir son nom décliné en adjectif dès l'Antiquité tardive, où l'on voit souvent les pelagiani accompagnés de caelestiani ${ }^{59}$. Walter DUNPHY $(1994$, p. 36) écrit même :

Célestius était si actif et si bien connu que son nom était invariablement cité dans les nombreuses condamnations à travers les années de la controverse et pendant un temps il était difficile de savoir si ce que nous connaissions en tant que pélagianisme n'aurait pas été en fait un "célestianisme". 60

40 Cette question nous emmènerait trop loin et nous éloignerait quelque peu de notre sujet principal mais l'on peut noter que Célestius avait bien des sujets de prédilection par rapport à Pélage qui insiste plus sur la notion de libre-arbitre que son disciple.

De plus, le passage de Célestius à Carthage n'a pas que des conséquences sur l'homme luimême et la diffusion de sa doctrine, mais aussi sur la population de Carthage dont une partie a bien accueilli les discours. Ses succès forment ce qu'on peut appeler la mémoire vivante du passage de Célestius à Carthage, ce dont se plaint particulièrement Augustin puisqu'il a dû déployer une énergie supplémentaire pour combattre les idées que Célestius avait semées. Il écrit ainsi en 414 :

Multa de his quaestionibus in aliis nostris opusculis et ecclesiasticis sermonibus diximus, quoniam fuerunt etiam apud nos quidam, qui, ubicumque poterant, haec sui erroris noua semina spargerent.

(Aug., Ep. 157, 3, 22)

Sur beaucoup de ces questions, nous nous sommes expliqués dans nos autres ouvrages et dans nos sermons dans les églises, car il s'en est trouvé, même chez nous, pour répandre partout où ils pouvaient les germes nouveaux de leur hérésie.

Combien de temps les « germes » du pélagianisme disséminés par Célestius survécurentils après son passage? Nous ne pouvons pas le savoir exactement. En 427, soit neuf ans après la condamnation définitive du pélagianisme, Augustin adresse encore des avertissements à un certain Vital de Carthage qui professe quelques idées pélagiennes ${ }^{61}$. 
Cependant, rien ne prouve que la contamination, pour reprendre un terme médical souvent utilisé pour décrire les "maux » que sont les hérésies, ait eu lieu à partir de la souche célestienne de 411. Si c'était toutefois le cas, le séjour de Célestius à Carthage, bien que bref, aurait eu des conséquences encore seize ans plus tard. Il n'en demeure pas moins que ce passage est tout à fait capital dans les enjeux doctrinaux du début du ve siècle.

Enfin, le corollaire de ce passage est bien sûr la condamnation de Célestius à la réunion de Carthage, conférant à cet événement une mémoire sur un courant d'idées, et non plus seulement sur un lieu géographique.

\section{La « condamnation » de Carthage : un acte fondateur pour les antipélagiens}

$\mathrm{Du}$ fait de l'incertitude dans laquelle nous laissent les sources, nous ne pouvons qu'employer le mot vague de " condamnation». Quelle que soit la gradation de celle-ci, elle n'a en tout cas pas réglé une fois pour toutes le sort des pélagiens puisque ceux-ci continuent de répandre leurs idées. Ils sont ainsi présents en Sicile (Ep. 156 et 157) et surtout en Orient: Célestius obtient la prêtrise à Éphèse en 415, tandis que Pélage, la même année, est absous des accusations portées contre lui par des Occidentaux, au concile de Diospolis. Fait particulièrement intéressant dans ce procès : la condamnation de Carthage a été invoquée comme jurisprudence et les accusateurs de Pélage ont voulu l'utiliser comme une preuve de sa propre culpabilité ${ }^{62}$. Pélage n'en est pas moins absous par les évêques orientaux et cette décision relance la machine d'opposition africaine. Celle-ci avait, en effet, toute confiance dans la sûreté et la fermeté du jugement rendu en 411, ce qui explique d'ailleurs certainement qu'Augustin n'ait pas été mis au courant de toute l'affaire à l'époque. À quoi bon? La situation avait été réglée une bonne fois pour toutes.

45 Le jugement de Diospolis vient donc contredire le précédent africain. Le clergé de cette région se sent ainsi obligé de justifier son action de 411 et de trouver la faille de celle de 415 - poussé par des raisons doctrinales, ou peut-être par un sentiment de rivalité provinciale.

Ainsi, deux conciles sont organisés en Afrique, l'un à Milev en Numidie, l'autre à Carthage en 416, alors que les actes de Diospolis ne sont pas encore connus dans le détail. Les mesures prises par les Africains sont contenues dans la Lettre 175 du corpus augustinien. Adressée au pape Innocent par les évêques de Proconsulaire réunis au concile de Carthage, elle vise à obtenir de l'évêque romain une caution supplémentaire pour confirmer les résolutions africaines afin de les rendre incontestables par d'autres clergés. On peut ainsi y lire :

Vnde factum est, ut recensendum peteremus, quid ante ferme quinquennium super Celestii nomine hic apud escclesiam Carthaginensem fuerit agitatum. Quo recitato, sicut ex subditis aduertere poterit sanctitas tua, quamuis iudicatio manifesta constaret, quia illo tempore episcopali iudicio excisum hoc tantum uulnus ab ecclesia uideretur, nihilo minus tamen id communi deliberatione censuimus, ut huius modi persuasionis auctores, quamuis et ad presbyterium idem Caelestius postea peruenisse dicatur, nisi haec apertissime anathemauerint, ipsos anathemari oporteat.

$($ Ep. 175, 1)

D'où il résulte que nous cherchions à passer en revue ce qui a été fait dans l'Église de Carthage, il y a à peu près cinq ans, sur Célestius. Cela ayant été produit comme 
votre sainteté pourra le remarquer d'après ce qui a été mis à votre disposition : quoiqu'un jugement manifeste soit établi disant que cette si grande blessure semblait avoir été enlevée par l'Église à la suite d'un jugement épiscopal, néanmoins nous avons jugé à la suite d'une délibération commune qu'il fallait que les auteurs d'une doctrine de cette sorte soient précisément anathématisés s'ils ne l'ont pas eux-mêmes anathématisée ouvertement, quoiqu'on dise que le même Célestius est parvenu par la suite à la prêtrise. plusieurs égards. Il marque le début de la phase active d'expansion du pélagianisme et de l'affirmation de Célestius dans le groupe des pélagiens. Il est aussi associé au début de la lutte antipélagienne avec la réunion de Carthage de 411. E. D. LAVENDER (1992, p. 2) va jusqu'à écrire que «ce qui était auparavant une orthodoxie locale indifférenciée devint une hérésie seulement après [l']arrivée [de Célestius] à Carthage ${ }^{64}$ ».

Certes, Célestius n'était pas seul à Carthage mais accompagné de disciples de Pélage ou d'amis. Ces autres personnes ont bien sûr joué un rôle dans la diffusion des idées nouvelles. Cependant la postérité n'a retenu que le nom de Célestius, qui endosse ainsi le rôle principal du changement d'état du pélagianisme et qui apparaît comme un chef de file incontournable.

Voici donc un passage qui aurait pu être sans conséquence - Augustin n'est pas au courant de ces événements avant plusieurs semaines, mois, voire années selon les historiens, de la prédication et de la condamnation de Célestius - et qui eut finalement un très grand rayonnement dans l'espace et le temps. 


\section{BIBLIOGRAPHIE}

\section{Abréviations}

Academia = Academia. Journal of the Nanzan Academic Society. Humanities and Social Sciences.

AugStud = Augustinian Studies. Villanova (Pa.), Villanova University Pr.

$B A=$ Bibliothèque augustinienne. Euvres de saint Augustin, Paris, depuis 1936.

$C S E L=$ Corpus scriptorum ecclesiasticorum latinorum (dit « Corpus de Vienne »), Vienne, depuis 1866.

DHGE = Dictionnaire d'histoire et de géographie ecclésiastiques.

JThS = Journal of theological studies, Oxford, Clarendon Pr.

$P C B E=$ Prosopographie chrétienne du Bas-Empire.

PL = Patrologiae cursus completus. Series Latina, éd. J.-P. Migne, Paris, 1844-1855.

REAug = Revue des études augustiniennes, devenue en 2004 Revue d'études augustiniennes et patristiques.

SC = Sources chrétiennes, Paris, depuis 1942.

$V C h r=$ Vigiliae christianae $:$ a review early Christian life and languages, Brill, Leiden.

\section{Sources}

Augustin d'Hippone (= Aug.)

Contra Iulianum (= C. Iul.), PL 44, Paris, 1865, 640-874.

De diuersis quaestionibus ad Simplicianum, BA 10, int., trad., notes par G. Bardy, J.-A. Beckaert, J. Boutet, Paris, 1952.

De gratia Christi et de peccato originali (= Gr. et pecc. or.), BA 22, int., trad., notes par H. Chirat et J.

Plagnieux, Paris, 1975.

De peccatorum meritis et remissione et de baptismo paruulorum (= Pecc. mer.), De spiritu et littera (= Spir. et litt.), CSEL 60, éd. par C.-F. Vrba, J. Zycha, Vienne, 1913.

Epistulae (= Ep.), éd. d'Al. Goldbacher, CSEL, Vienne, 44 (1904), 57 (1911) et 58 (1923).

La crise pélagienne I, Epistula ad Hilarium Syracusanum (= Ep. 156 et 157), De perfectione iustitiae hominis (Perf. iust.), De natura et gratia (Nat. et gr.), De gestis Pelagii (= Gest. Pel.), BA 21, int., trad., notes par G. de Plinval et J. de la Tullaye, Paris, 1966.

Premières polémiques contre Julien, Contra duas epistulas pelagianorum (=C.ep. Pel), BA 23, int., trad. et notes par F.-J. Thonnard, E. Bleuzen, A.-C. de Veer, Paris, 1974.

Retractationes (= Retr.), BA 12, int., éd., trad., notes par G. Bardy, Paris, 1959.

De Haeresibus ad Quoduultdeum, CCSL 46, éd. par R. Vander Plaetse et C. Beukers, Turnhout, 1969. 
Arnobe le Jeune, Praedestinatus qui dicitur, CCSL 25, éd. par Fr. Gori, 2001.

Gérontius, Vie de sainte Mélanie, SC 90, int., trad, notes par Denys Gorce, 1962.

Jérôme (= Jér.), Lettres (= Ep.), int., éd., trad, notes par Jérôme Labourt, Paris, 1949.

Marius Mercator (= Mar. Mer.)

Commonitorium super nomine Caelestii (= Comm. nom. Cael.) , Coll. Palatina 36, ACO I, 5, 1, p. 65-70.

Commonitorium aduersum haeresim Pelagii et Caelstii uel etiam scripta Iuliani (= Comm. adu. haer. Pel.), Coll. Palatina 13, ACO I, 5, 1, p. 5-23.

Orose, Liber apologeticus contre Pelagianos, CSEL 5, éd. par C. Zangemeister, 1882, p. 601-664.

Paulin de Milan, Vita Ambrosii, éd. A.A.R. Bastiaensen, Milan, 1975.

Paulin de Nole, Epistulae, CSEL 29, éd. par G. de Hartel, Vienne, 1894.

Vincent de Lérins, Commonitorium, CCSL 64, éd. par R. Demeulenaere, Turnhout, 1985, p. 147-195.

\section{Bibliographie générale}

ARNAUD P. (2005), Les routes de la navigation antique, Paris.

BROWN P. (2001 pour la trad. fr.), La vie de Saint Augustin, Paris.

BROWN P. (1970), « The patrons of Pelagius. The Roman aristocracy between West and East ", JThS XXI, p. 56-72.

DELAROCHE B. (1995), « La datation du De peccatorum meritis et remissione », REAug 41, p. 37-57. DODARo R. (2000), « Note on the Carthaginian Debate over Sinlessness, A.D. 411-412 (Augustine, pecc. mer. 2.7.8-16.25)», Augustinianum 40, p. 187-202.

DUNPHY W. (1994), «Caelestius : A Preliminary Investigation », Academia 60, p. 33-59.

DUNPHY W. (1995), « The Writings of Caelestius », Academia 61, p. 25-47.

DUNPHY W. (1997), « Pelagius or Caelestius ? An Unidentified Text », Academia 65, p. 147-165.

DUNPHY W. (2005), « A lost year : Pelagianism in Carthage, 411 A.D. », Augustinianum 45, p. 389-466.

DUNPHY W. (2009), « Rufinus the Syrian : myth and reality », Augustiniana 59, p. 79-157.

DUVAL Y.-M. (2001), « Pélage en son temps : Données chronologiques nouvelles pour une présentation nouvelle ", Wiles M. F. Yarnold E. J. éd., Studia Patristica 38 : St. Augustine and his Opponents. Other Latin Writers, Louvain, p. 95-118.

HONNAY G. (1994), « Caelestius, discipulus Pelagii », Augustiniana 44, p. 271-302.

Koopmans J. H. (1954), « Augustine's First Contact with Pelagius and the Dating of the Condemnation of Caelestius at Carthago », Vchr 8, p. 149-153.

LANCEL S. (1999), Saint Augustin, Paris.

LAVENDER E. D. (1992), The Development of Pelagius' Thought within a Late Fourth Century Ascetic Movement in Rome, Ann Arbor.

NUVolone F. G. (1986), « Pélage et le pélagianisme », Dictionnaire de spiritualité, XII, Paris, col. 2889-2942.

PERLER O. (1969), Les voyages de saint Augustin, Paris, p. 280-286. 
PIETRI Ch. et L. dir. (1995), Histoire du christianisme 2. Naissance d'une chrétienté (250-430), Paris.

PIETRI Ch. et L. dir. (1999-2000), PCBE, Volume 2 : Prosopographie de l'Italie chrétienne (313-604), Paris, 1999-2000.

REFOULÉ F. (1963), « Datation du premier concile de Carthage contre les Pélagiens et du Libellus fidei de Rufin » REAug 9, p. 41-49.

SALAMITO J.-M. (2005), Les virtuoses et la multitude, Aspects sociaux de la controverse entre Augustin et

les pélagiens, Grenoble.

WERMELINGER O. (1975), Rom und Pelagius : Die theologische Position der römischen Bischöfe im

pelagianischen Streit in den Jahren 411-432, Stuttgart.

\section{NOTES}

1. Il n'est que de citer la correspondance d'Augustin sur le sujet avec Marcellinus (Ep. 143). Le docteur d'Hippone n'ayant pas réussi à trouver de réponse satisfaisante à la question soulevée par son correspondant, il s'adresse à Jérôme (Ep. 166 aussi appelée De origine animae) en quête de réponses. Mais lorsque le solitaire de Bethléem reçoit cette lettre, la controverse pélagienne bat son plein en Orient et il ne trouve plus l'occasion d'y répondre.

2. Le mot pélagianisme est une construction postérieure à l'Antiquité et n'a donc jamais été connu des acteurs de la controverse pélagienne (contrairement à l'adjectif "pelagianus»). Cependant, il sera utilisé dans cet article par commodité, et à l'ombre de l'autorité de mes prédécesseurs même si les recherches récentes critiquent de plus en plus ce terme générique qui cacherait plusieurs tendances bien distinctes et qui fait la part belle à Pélage en occultant les autres penseurs qui en sont proches - notamment Célestius (voir les références note 29).

3. Qualifié de la sorte dans plusieurs sources (Augustin, Ep. 186, 1 ; Mar. Mer., Comm. adu. haer. Pel, ACO I, v, 1, p. 5).

4. Nous signalons que des doutes ont été émis sur l'identification de Célestius comme principal responsable du déclenchement du débat carthaginois notamment par Robert DODARO (2000) qui parle cependant d'un "consensus » des historiens sur la "position centrale » que Célestius a occupée (p.197), consensus que nous suivons. Et même si Célestius n'avait effectivement joué qu'un rôle secondaire dans le fait de répandre le pélagianisme à Carthage, il nous semble que les conséquences de sa condamnation - qui, elle, est sûre - dans la suite de l'histoire pélagienne justifie l'intérêt que nous portons à cet épisode.

5. On ne connaît pas la date exacte de son arrivée en Afrique, mais nous proposons une hypothèse un peu plus loin.

6. Mar. Mer., Comm. nom. Cael, ACO I, v, 1, p. 66 : «Un certain Célestius, disciple et auditeur de Pélage depuis un peu plus de vingt ans, sortit de la ville de Rome et vint dans la métropole de toute l'Afrique, Carthage. » ; Caelestius quidam (...) ante uiginti plus minus annos discipulus et auditor Pelagii egressus ex urbe Romana Carthaginem Africae totius metroplim uenit.

7. Pour une synthèse des débats antérieurs à 411, on se reportera à l'article d'Yves-Marie DUVAL (2001, p. 100-101).

8. Pour un portrait détaillé, on se reportera à la notice prosopographique à PIETRI (1999-2000, p. 357-375).

9. Aug. C. ep. Pel. II, 3, 5 : « in homine acerrimi ingenii »

10. Mar. Mer., Comm. adu. haer. Pel, ACO, I, v, 1, p. 6 : «Célestius s'attacha à ce Pélage, il était de naissance noble certes et avocat de cette époque-là mais sorti eunuque du ventre de sa mère par un défaut de la nature. Celui-ci, formé par Pélage que j'ai mentionné avant, se pénétra sans aucun 
mélange de cette conception très impie et rendit beaucoup de gens participants et complices de sa folie grâce à son éloquence incroyable, et il osa ouvertement et en public propager de tous côtés à travers les peuples la conception de ce même homme par les idées exprimées par ces paroles "; Huic Pelagio adhaesit Caelestius, nobilis natu quidem et illius temporis auditorialis scholasticus, sed naturae uitio eunuchus matris utero editus. Hic a Pelagio praedicto institutus, sensum istum impiissimum meracius inbibit ac multos incredibili loquacitate amentiae huius suae participes et conplices fecit aususque palam publiceque his uerborum sententiis eiusdem sensum passim disseminare per populos.

11. Célestius semble bien avoir côtoyé Pammachius à Rome selon ses propos tenus pendant la réunion de Carthage en 411 et rapportés dans Aug., Gr. et pecc. or., III. 3 : «Le vénérable prêtre Rufin qui demeura à Rome en compagnie du vénérable Pammachius. Je l'ai entendu dire qu'il n'y a pas transmission de péché. » : Sanctus presbyter Rufinus, Romae qui mansit cum sancto Pammachio; ego audiui illum dicentem quia tradux peccati non sit.

12. D'autant plus que nous savons que l'un des serviteurs de Pinianus, Timase (Aug. Ep. 126, 6) était aussi l'un des disciples les plus proches de Pélage, comme nous l'apprend le Nat. et gr. d'Augustin $(1,1)$.

13. Aucune mention de Pélage n'est faite dans la Vie de sainte Mélanie (SC 90), mais on ne doit pas s'en étonner, puisqu'elle est postérieure à la condamnation ecclésiastique du pélagianisme.

14. On reconstitue cet itinéraire à partir de la Vie de sainte Mélanie par Gérontius. On y trouve en effet (19) : «Tandis qu'ils faisaient voile de la Sicile vers le très saint évêque Paulin, chez qui ils s'étaient déjà retirés au début ", ce qui suppose un premier voyage de Rome vers Nole, puis un second vers la Sicile, et enfin une tentative échouée de revenir à Nole avant de faire voile vers l'Afrique.

15. Cf. Aug, Gest. Pel. 22, 46 : « Mais, après être arrivé en Afrique, il fut reçu en mon absence sur la côte d'Hippone »: Postea uero quam in Africam uenit, me absente, nostro, id est Hipponensi litore exceptus est.

16. Plusieurs sources le confirment, voir entre autres : Gerontius, Vie de sainte Mélanie 21 : «La ville du très bienheureux évêque Alypius, nommée Thagaste, était petite et très pauvre. C'est elle que les bienheureux choisirent pour y demeurer, en raison surtout de la présence de ce saint personnage ».

17. Cf. Aug., Ep. 124.

18. Par exemple, la correspondance de Paulin de Nole le lie à la fois à Alypius (ex : Ep. 3), Augustin (ex : Ep. 4), Pammachius (ex : Ep. 13), Pélage (cf. Aug., Ep. 186, 1 et Gr. et pecc. or., 35, 38).

19. Augustin écrit dans le Gr. et pecc. or., 22, 46 : « Mais, après être arrivé en Afrique, [Pélage] fut reçu en mon absence sur la côte d'Hippone où, comme je l'ai appris par des amis, rien de tel ne fut entendu de sa part, car il partit plus vite que l'on ne pensait. Peu après, autant que je m'en souvienne, j'ai aperçu une fois ou deux son visage à Carthage, au moment où j'étais extrêmement occupé par l'organisation de la conférence que nous étions sur le point d'avoir avec les hérétiques donatistes ; mais, lui, se hâta de partir outre-mer. "; Postea uero quam in Africam uenit, me absente, nostro, id est Hipponensi litore exceptus est, ubi omnino, sicut comperi a nostris, nihil ab illo huius modi auditum est, quia et citius quam putabatur, inde profectus est. Postmodum eius faciem Carthagine, quantum recolo, semel uel iterum uidi, quando cura collationis, quam cum haereticis Donatistis habituri eramus, occupatissimus fui ; ille uero etiam ad transmarina properauit.

20. Voir l'Ep. 157 et le Perf. iust. : Augustin a été informé par des « frères » que Célestius a séjourné en Sicile et y a fait de nombreux adeptes.

21. Cf. Aug, Gest. Pel. 22, 46 : « j'ai connu le nom de Pélage tout d'abord quand il était au loin établi à Rome, entouré de grands éloges » : prius absentis et Romae constituti Pelagii nomen cum magna eius laude cognoui.

22. Aug. Gr. et pecc. or., 22, 46: "mais, lui, se hâta de partir outre-mer »; ille uero etiam ad transmarina properauit. 
23. Ainsi J. H. Koopmans (1954) concluait-il du départ précipité de Pélage que la condamnation de Célestius avait déjà eu lieu et que celui-ci voulait en éviter les représailles. Nous ne retenons cependant pas la datation haute de la réunion de Carthage de cet auteur.

24. On se reportera par exemple au bilan que dresse Augustin de ce fait dans l'Ep. 157, 3, 22.

25. "Caelestius, nobilis natu, could (rightly or not) consider himself to be a representative member of the Roman nobility then present in unusual numbers in Carthage (...). A person of their rank was being accused of nothing less serious than heresy by a 'mere' deacon of Milan, on a subject that was - at least in Roman circles - undefined and open to discussion, before a 'mere' provincial bishop, while the bishop of Rome had seen no need to take a stance on the question in debate! ».

26. Par exemple en IX. 9 : eos; sentiunt etc.

27. Aug., Gest. Pel., 11, 25 : Marcellinum eos cotidie disputatores molestissimos patiebatur. On pourra nous objecter que c'est ici une référence à la réunion épiscopale réunie contre Célestius et non plus aux groupes de discussion restreints. Si l'on ne peut prouver définitivement que ce n'est pas le cas, du moins peut-on s'interroger sur le fait que ce soit Marcellinus qui subit les assauts des pélagiens et non plutôt Aurelius de Carthage qui présida l'assemblée des évêques réunie contre Célestius.

28. Aug., Spir. et litt., 35, 61 : sed tam uehementem, tam intentam uigilemque conflictationem.

29. Comme nous l'avons déjà signalé, aucun des noms des acteurs du débat de Carthage n'est mentionné (cf. DODARO 2000, déjà cité). On tentera d'expliquer ce fait un peu plus loin. On s'appuie donc sur les sources postérieures présentées dans ce présent article pour identifier le personnage principal de cet épisode, Célestius.

30. Les études pélagiennes tendent aujourd'hui à mettre l'accent sur les différences idéologiques entre Pélage et Célestius qui rendraient obsolète le mot " pélagianisme ", d'où l'importance de bien différencier les écrits de Pélage de ceux de Célestius. Cf. par exemple : DUNPHY 1997; DODARO 2000 ; SALAMITO 2005, p. 15, 16.

31. Arnobe le Jeune (?), Praedestinatus : Caelestiu[s] qui contra traducem peccati primus scripsit.

32. Cf. par exemple Aug, Contra Iulianum, I, 19.

33. L'écrit n.6 selon la numérotation de Flavio NUVOLONE (1986, col. 2891).

34. Aug., Pecc. mer., I, 24, 34 : Optime punici christiani baptismum ipsum nihil aliud quam salutem.

35. Augustin ne mentionne les novateurs que par la troisième personne du pluriel. Il parle cependant de l'opinion différente des Africains sous-entendant que les adversaires ne sont pas Africains.

36. Augustin se dit «nouitate permotus » (Aug., Pecc. mer., VI, 12).

37. Ainsi que biographe du célèbre évêque du lieu, Ambroise.

38. Cf. Mar. Mer., Comm. nom. Cael., ACO I, v, 1, p. 66, 1. 4, 5 : Célestius est accusé par le libelle d'un certain Paulin, diacre de l'évêque de Milan Ambroise de bien heureuse mémoire » : Caelestius (...) per libellum a quodam Paulino diacono sanctae memoriae Ambrosii Mediolanensis episcopi est accusatus.

39. Pensons ainsi à Simplicianus, prêtre de Milan qui avait fait appel à Augustin pour résoudre des questions portant sur des sujets aux thématiques proches des pélagiens. Cf. De diuersis quaestionibus ad Simplicianum.

40. Paulin de Milan, Vita Ambrosii 10.

41. «Man kann sich des Eindrucks nicht erwehren, dass die Kläger nur die vorgeschobenen Figuren sind, die sich aber durch die eigentlichen Drahtzieher voll gedeckt wissen. »

42. Ibid.: "Bezeichnenderweise haben in der pelagianischen Kontroverse ausserhalb Roms immer Emigranten geklagt ». On peut citer ainsi : Paulin de Milan à Carthage ; Lazare et Héros, deux évêques gaulois à Diospolis (Palestine); Marius Mercator, un Italien, à Constantinople.

43. Cf. Entre autres : J. H. KOOPMANs (1954); Walter DUNPHY (2005).

44. Cf. Othmar PERLER (1969), p. 293 : « le sermon $296 »$. 
45. Ibid: "c'est la nuit du 13 au 14 septembre [411] qu'il prêcha [à Carthage] l'Ennaratio in Psalmum $72 »$.

46. Utilisé par J. H. KOOPMANS (1954, p. 153) mais laissé de côté par exemple par François REFoUlé (1963) ou Bruno DELAROCHE (1995) qui sont d'accord pour le dater des environs d'octobrenovembre 411 même si le premier va jusqu'à décembre 411, ce qui n'est pas possible pour nous, à la suite du second.

47. Si l'on considère que le point d'arrivée est Éphèse par exemple, en partant de Carthage.

48. Mar. Mer., Comm. nom. Cael., ACO I, v, 1, p. 66, 1. 21 : Caelestius (...) frequenter auditus est.

49. Aug., Pecc. mer., I, 34, 63 : « Aussi bien, quand il s'agit du baptême des petits enfants, afin que celui-ci leur soit nécessaire, ils admettent que ceux-ci de même ont besoin de rédemption, comme on peut le lire dans un court écrit de l'un d'entre eux, qui ne veut cependant pas s'expliquer dedans plus ouvertement sur la rémission des péchés »: Proinde quod adtinet ad baptismum paruulorum, ut eis sit necessarius, redemptionem etiam ipsis opus esse concedunt, sicut cuiusdam eorum libello breuissimo continetur, qui tamen ibi remissionem alicuius peccati apertius esprimere noluit.

50. Ibid.

51. Orose, Liber apologeticus, CSEL 5, 3, 4 p. 607 : « conuictum »; « confessum »; 4, 5, p. 608 : Caelestio Africana synodus detestata est.

52. Aug., Retr. II, 33, 60 : « Dès ce moment, son disciple Célestius avait mérité l'excommunication à cause de pareilles affirmations, à Carthage, de la part d'un tribunal épiscopal dont je n'ai pas fait partie »: Caelestius uero, discipulus eius, iam propter tales assertiones apud Carthaginem in episcopali iudicio, ubi ego non interfui, excommunicationem meruerat.

53. Mar. Mer., Comm. nom. Cael., ACO, I, v, 1, p. 66, 1. 21, 22 : ecclesiastica communione priuatus est.

54. Pour une synthèse des avis sur ce sujet, voir Guido HonNAY (1994, p. 277 et en particulier la note 43$)$.

55. On a déjà cité Mar. Mer. Comm. nom. Cael., ACO I, v, 1, p. 66, 1. 22-25 ; voir aussi Orose, Liber apologeticus, CSEL 5, 3, 4 p. 607 : Célestius a été « entendu, confondu, a avoué et a fui hors de l'Afrique » : auditum, conuictum, confessum (...) ex Africa profugisse. La construction de la phrase semble bien indiquer que les actions s'enchaînent immédiatement dans le temps.

56. Mar. Mer., Comm. nom. Cael., ACO, I, v, 1, p. 66, 1. 22 : a qua sententia ad Romani episcopi examen credidit appellandum.

57. Comme le demandait Walter DUNPHY (1995, p. 36) : «The name of Caelestius emerges throughout the debate. We have reference to Caelestians as well to Pelagians. Yet the teaching of Caelestius has been given little attention. "

58. Jérôme affirme même en 413 que Célestius n'est plus le disciple de Pélage mais bien un hérésiarque, Ep. 133, 5: «L'un de ses disciples, ou plutôt maintenant un maître et chef de toute l'armée ». Il parle même en 419 de « haeresis Caelestina »; Unus discipulorum eius, imo iam magister et totius ductor exercitus (Ep. 143, 1).

59. Cf. par exemple Aug. C. ep. Pel., II, 5 ou De haeresibus 41.

60. "He was so active and well known that his name was invariably mentionned in the various condemnation through the years of the crisis and for a time it was not clear of what we have come to know as Pelagianism should not in fact have become known as Caelestianism ».

61. Cf. Aug, Ep. 217.

62. Aug., Gest. Pel., 35, 62 : «Comme cette hérésie abusait de nombreuses personnes et que, sans les abuser, elle troublait nos frères, un nommé Célestius qui partageait ces opinions fut traduit devant le tribunal de l'Église de Carthage et condamné par le verdict des évêques. Puis, quelques années plus tard, comme cette hérésie était imputée à Pélage qui passait pour le maître de Célestius, Pélage dut également se rendre devant un tribunal épiscopal. » : Ista haeresis cum plurimos decepisset et fratres, quos non deceperat, conturbaret, Caelestius quidam talia sentiens ad 
iudicium Carthaginensis ecclesiae perductus episcoporum sententia condemnatus est. Deinde post aliquot annos Pelagio, qui magister eius perhiberetur, cum ista haeresis fuisset obiecta, ad episcopale iudicium.

63. C. Iul., III, 1, 5: Qui debellauit apud Carthaginem Caelestium, quando illic ipse non eram.

64. «What was previously undifferenciated local orthodoxy became heresy only after it arrived in Carthage ».

\section{RÉSUMÉS}

Durant le printemps-été 411, la ville de Carthage accueille Pélage et son plus proche disciple, Célestius, personnage encore inconnu. Le premier se hâte de quitter l'Afrique pour l'Orient contrairement au second, qui profite de son séjour dans la capitale africaine pour répandre, pour la première fois en dehors de petits cercles italiens, leur doctrine. Cet épisode marque profondément la vie de Célestius, mais surtout l'histoire du pélagianisme, qui entre alors dans une phase d'expansion active inédite mais connaît aussi une vague d'oppositions sans précédent.

During Spring and Summer 411, Pelagius and his closest pupil, the then-unknown Caelestius, arrived at Carthage. The former quickly left Africa for the East, while the latter took advantage of his stay in the African capital to spread their doctrine - which, up to then, had been limited to the small Italian circles they used to live in. This episode deeply marked Caelestius's life and the history of Pelagianism, which then entered a phase of active expansion but also unleashed a wave of unprecedented opposition.

\section{INDEX}

Mots-clés : Augustin, hérésie, Pélage, Célestius, pélagianisme, voyage

Keywords : Augustine, heresy, Pelagius, Caelestius, pelagianism, travel

\section{AUTEUR}

\section{ZOHRA PICARD-MAWJI}

Centre « Méditerranée antique : civilisation et christianisme ancien » - UMR 8167 